\title{
Analysis of the retinal capillary plexus layers in a murine model with diabetic retinopathy: effect of intravitreal injection of human CD34 ${ }^{+}$bone marrow stem cells
}

\author{
Kong Wa Cheung ${ }^{1}$, Amirfarbod Yazdanyar ${ }^{1,2}$, Christian Dolf ${ }^{1}$, Whitney Cary ${ }^{3}$, \\ Nicholas Marsh-Armstrong ${ }^{1}$, Jan A. Nolta ${ }^{3}$, Susanna S. Park ${ }^{1}$ \\ ${ }^{1}$ Department of Ophthalmology \& Vision Science, University of California Davis Eye Center, Sacramento, CA, USA; ${ }^{2}$ Department of \\ Ophthalmology \& Visual Sciences, SUNY Upstate, Syracuse, NY, USA; ${ }^{3}$ Department of Internal Medicine, Stem Cell Program and Gene Therapy \\ Center, University of California Davis, Sacramento, CA, USA \\ Contributions: (I) Conception and design: SS Park; (II) Administrative support: N Marsh-Armstrong, JA Nolta, SS Park; (III) Provision of study \\ materials or patients: W Cary; (IV) Collection and assembly of data: KW Cheung, A Yazdanyar, C Dolf, W Cary, SS Park; (V) Data analysis and \\ interpretation: KW Cheung; (VI) Manuscript writing: All authors; (VII) Final approval of manuscript: All authors. \\ Correspondence to: Susanna S. Park, MD. PhD. Department of Ophthalmology \& Vision Science, University of California Davis Eye Center, $4860 \mathrm{Y}$ \\ St., Suite 2400, Sacramento, CA 95817, USA. Email: sscpark@ucdavis.edu.
}

Background: Diabetic retinopathy is a retinal vasculopathy involving all three retinal capillary plexus layers. Since human CD34 bone marrow stem cells (BMSCs) have the potential to promote revascularization of ischemic tissue, this study tests the hypothesis that intravitreal injection of human CD34 ${ }^{+}$BMSCs can have protective effects on all layers of the retinal vasculature in eyes with diabetic retinopathy.

Methods: Streptozotocin (STZ)-induced diabetic mice were injected intravitreally with 50,000 human $\mathrm{CD}^{+} 4^{+}$BMSCs or phosphate-buffered saline (PBS) into the right eye. Systemic immunosuppression with rapamycin and tacrolimus was started 5 days before the injection and maintained for study duration to prevent rejection of human cells. All mice were euthanized 4 weeks after intravitreal injection; both eyes were enucleated for retinal flat mount immunohistochemistry. The retinal vasculature was stained with Isolectin-GS-IB4. Confocal microscopy was used to image four circular areas of interest of retina, 1-mm diameter around the optic disc. Images of superficial, intermediate, and deep retinal capillary plexus layers within the areas of interest were obtained and analyzed using Image J software with the Vessel Analysis plugin to quantitate the retinal vascular density and vascular length density in the three plexus layers.

Results: Three distinct retinal capillary plexus layers were visualized and imaged using confocal microscopy. Eyes that received intravitreal injection of CD $34^{+}$BMSCs $(\mathrm{N}=9)$ had significantly higher vascular density and vascular length density in the superficial retinal capillary plexus when compared to the untreated contralateral eyes $(\mathrm{N}=9)$ or PBS treated control eyes $(\mathrm{N}=12$; $\mathrm{P}$ values $<0.05$ using ANOVA followed by post-hoc tests). For the intermediate and deep plexus layers, the difference was not statistically significant.

Conclusions: The protective effect of intravitreal injection of the human CD $34^{+} \mathrm{BMSCs}$ on the superficial retinal capillary plexus layers is demonstrated using confocal microscopy in this murine model of diabetic retinopathy.

Keywords: Confocal microscopy; diabetic retinopathy; human $\mathrm{CD}_{3} 4^{+}$stem cells; retinal flat-mount; retinal vascular plexus

Submitted May 14, 2020. Accepted for publication Sep 25, 2020.

doi: 10.21037/atm-20-3930

View this article at: https://dx.doi.org/10.21037/atm-20-3930 


\section{Introduction}

Diabetic retinopathy is a common complication of diabetes mellitus; it is the leading cause of blindness and low vision among working-aged adults aged $20-74$ years in the United States (1). It is characterized by progressive damage to the vasculature of the retina, eventually resulting in dysfunction of the retina and visual impairment that impacts the quality of life of the individual. Different treatments, such as intravitreal injection of drugs that inhibit vascular endothelial growth factor and retinal laser photocoagulation, are available to minimize the vision loss associated with complications of diabetic retinopathy, such as macular edema or retinal neovascularization. However, visual impairment from retinal ischemia and retinal dysfunction is irreversible.

Stem cells are being explored as a potential regenerative therapy for vision loss associated with diabetic retinopathy. Various stem cells have been explored for tissue regeneration for retinal disorders (2). In human bone marrow, there are $\mathrm{CD} 34^{+}$stem cells that include endothelial progenitor cells and hematopoietic stem cells (3). Human $\mathrm{CD} 34^{+}$bone marrow stem cells (BMSCs) are mobilized from the bone marrow into the circulation in response to tissue ischemia (4). They migrate to sites of tissue ischemia and integrate into the damaged vasculature for tissue regeneration and angiogenesis. Thus, the potential retinal vascular regenerative effects of these cells have been explored in animal models of retinal vasculopathy, including diabetic retinopathy (5).

Adult bone marrow is a major source of human CD $34^{+}$ stem cells. These stem cells appear to play an important role in vascular repair via multiple mechanisms $(6,7)$. There is preclinical evidence showing that these human CD $34^{+}$BMSCs can secrete proangiogenic factors and have paracrine trophic effects on endothelial cells and pericytes to promote angiogenesis $(4,8)$. Human CD $34^{+}$stem cells also contain endothelial progenitor cells that can directly engraft into damaged retinal vasculature (5).

By harvesting human CD $34^{+}$stem cells from bone marrow and directly injecting the cells into the vitreous cavity of the eye, we may maximize the regenerative potential of these cells on the retina. Preclinical studies have shown that intravitreal injection of human $\mathrm{CD} 34^{+}$cells from bone marrow or peripheral blood of healthy human donors results in rapid direct homing and integration of the human cells into the damaged portion of the retinal vasculature in a murine model of diabetic retinopathy or acute retinal ischemia-reperfusion injury $(5,9,10)$. Long-term retinal vascular integration of these human cells was noted in nonobese diabetic-severe combined immunodeficient (NODSCID) mice with acute retinal ischemia and reperfusion injury with no ocular or systemic safety concerns following intravitreal injection of human CD34 $4^{+}$BMSCs (9). More recently, we used a murine model of diabetic retinopathy to demonstrate molecular changes in the murine retina that regulate the pathogenesis of diabetic retinopathy following intravitreal injection of human CD $34^{+}$BMSCs (10). A potential protective effect of $\mathrm{CD} 34^{+}$cells on the overall retinal vasculature was noted in these murine eyes with diabetic retinopathy but detailed analysis of the different layers of the retinal vasculature was not performed.

The retinal vasculature consists of three distinct capillary plexus layers: superficial, intermediate, and deep plexus. In eyes with diabetic retinopathy, retinal capillary changes in all three plexus layers have been described (11-13). Vascular changes in different plexus layers have been associated with the progression of retinopathy, retinal ischemia, and vision loss. To access the retinal vascular regenerative potential of intravitreally injected human CD $34^{+}$BMSC, it would be important to know whether these stem cells can have protective effects on the deeper retinal capillaries as well as the superficial vessels since immunohistochemical studies in murine eyes with diabetic retinopathy identified these human cells only in the superficial retinal layer following intravitreal injection (10). In this study, we used confocal microscopy analysis of retinal flat mount of murine eyes with diabetic retinopathy to test the hypothesis that intravitreal injection of human $\mathrm{CD} 34^{+}$BMSCs has protective effects on all three retinal capillary plexus layers. The murine model used in this study is the most commonly used model of diabetic retinopathy since it is high reproducible and exhibit many changes associated with diabetic retinopathy in human eyes (10). We present the following article in accordance to the ARRIVE reporting checklist (available at https://dx.doi.org/10.21037/atm-203930).

\section{Methods}

\section{Animal model and retinal flat mount immunobistochemistry}

All experiments in this study were conducted in accordance with the Association for Research in Vision and Ophthalmology (ARVO) Statement for the Use of Animals 
in Ophthalmic and Vision Research and a protocol approved by the Institutional Animal Care and Use Committee at the University of California Davis mandated by the Animal Welfare Act and the Healthy Research Extension Act (Protocol \#20162).

The retinal flat mounts from murine eyes with diabetic retinopathy were obtained as part of our prior study evaluating the effect of intravitreal injection of human CD $34^{+}$BMSC in murine eyes with diabetic retinopathy (10). Thus, details regarding animal experimentation have been described previously. Briefly, male streptozotocin (STZ)-induced diabetic mice were obtained commercially (C57BL/6J; Jackson Laboratories, Sacramento, CA, USA). Six-week-old C57BL/6J mice received 5 daily intraperitoneal injections of STZ $(50 \mathrm{mg} / \mathrm{kg})$ for 1 week and the development of diabetes was confirmed when blood sugar measured $>250 \mathrm{mg} / \mathrm{dL}$ on day 7 (14). The mice were then shipped to the study center vivarium where they were maintained in the diabetic state for 5 additional months till early development of diabetic retinopathy. The welfare of the animals was assessed daily for the duration of the study. Using fluorescein angiography, early retinal vascular changes consistent with diabetic retinopathy were noted to develop in these mice between 5 and 6 months after induction of diabetes (10).

Continuous systemic immunosuppression was induced in mice by subcutaneous placements of Alzet micro-osmotic pumps (model 1004; Durect Corporation, Cupertino, CA, USA) preloaded with rapamycin and tacrolimus (FK506) to release each drug at a constant rate of $1 \mu \mathrm{g} / \mathrm{g} /$ day. This was done 5 days before intravitreal injection to avoid rejection of human cells similar to our previous work (15).

The mice were randomly divided into two groups. In one group ( $\mathrm{n}=9$ ), 50,000 human CD34 $4^{+}$BMSCs labeled with enhanced green fluorescent proteins (EGFP) were injected intravitreally into the right eye $(1 \mu \mathrm{L})$. The human bone marrow obtained commercially from a healthy donor was the source of the $\mathrm{CD} 34^{+}$cells. The CD $34^{+}$BMSCs were harvested from bone marrow using a Ficoll gradient separation and magnetic beads and labeled with EGFP using a lentiviral vector as previously described (15). In the second group $(\mathrm{n}=12)$, phosphate-buffered saline (PBS; $1 \mu \mathrm{L})$ was injected into the vitreous of the right eye as a control. Systemic immunosuppression was maintained for the study duration of 4 weeks. No sample size calculation was performed for this study since this is a follow-up study of a prior study showing a significant effect of $\mathrm{CD} 34^{+}$cells (10).
At 4 weeks following intravitreal injection, the mice were euthanized by asphyxiation with gaseous $\mathrm{CO}_{2}$ in a closed chamber and the eyes were removed for fixation in $4 \%$ paraformaldehyde in PBS at $4{ }^{\circ} \mathrm{C}$ for 1 hour as previously described (10). The retina was dissected, flatmounted, and fixed under coverslips overnight in cold paraformaldehyde (16). Retinal samples were then stored at $-20{ }^{\circ} \mathrm{C}$ in $100 \%$ methanol after graded series of washes in PBS, 33\%, 66\%, and 100\% methanol. The reverse graded series of methanol was used to rehydrate the retinal samples before immunostaining. The samples were incubated for 24 hours at $4{ }^{\circ} \mathrm{C}$ in blocking solution $(5 \%$ normal donkey serum in PBS with $0.3 \%$ Triton $\mathrm{X}-100$ ) and the blood vessels on the retinal samples were stained with 1:250 Alexa Fluor-647 conjugated Isolectin-GS-IB4 from Griffonia simplicifolia (Invitrogen I32450, lot 1874784). All the samples were then mounted on a slide using Aqua Poly/ Mount (Polysciences) after a PBS wash.

\section{Confocal microscopy imaging}

Confocal microscopy was performed using a laser-equipped Olympus FV 1000 Confocal microscope (Olympus America Inc., Center Valley, PA, USA). Laser with 647-nm wavelength and $25 \%$ power output was used in image acquisition. The optic disc was used as a landmark for each retinal sample. Four circular areas of interest of $1 \mathrm{~mm}$ in diameter (up, down, left, right) centered around the edge of the optic disc were capture under the $10 \times$ objective (Figure 1). For each area of interest, a stack of 10 to 12 1024-pixel $\times 1024$-pixel microscopic scans was obtained along the $\mathrm{Z}$-axis using a bi-directional scan mode set at speed of $8.0 \mu \mathrm{s} /$ pixel with a pixel size of $0.1 \mu \mathrm{m} /$ pixel. Each stacked scan was separated by $4.4 \mu \mathrm{m}$ in the Z-direction.

The scanned stacks for each area of interest were reviewed to identify the superficial, intermediate, and deep retinal capillary plexus layers. By scrolling through the $\mathrm{Z}$-axis, the three interconnected capillary plexus layers were identified. For each stack of scans, the scanned layer with the highest vascular density for each of the superficial, intermediate, and deep capillary plexuses was selected for vascular density analysis. Scans with poor image acquisition signal (signal-to-noise ratio $<3$ ) were excluded from the analysis. Furthermore, scans with irregularity in the image from tissue folds or suboptimal immunohistochemical staining were excluded from data analysis if the irregularity involved $50 \%$ or more the scanned image area. 


\section{Page 4 of 12}

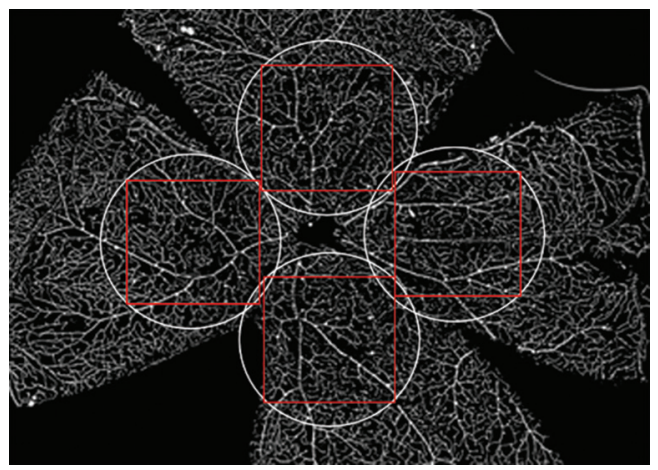

Figure 1 Four retinal areas of interest centered around the optic disc imaged in each retina using confocal microscopy. White circles indicate the fields of view under the $10 \times$ objective. Red squares indicate the areas captured by the scanner of the microscope. Each square is recorded with 1024-pixel by 1024-pixel in the XY-axis. Ten to twelve scans were taken in the $\mathrm{Z}$-axis $4.4 \mu \mathrm{m}$ apart for each red square.

\section{Retinal vascular density and vascular length density analysis}

The primary outcome measures were retinal vascular density and vascular length density as quantitated using ImageJ software (https://imagej.nih.gov, National Institute of Health, Bethesda, Maryland, USA) with the Vessel Analysis plugin as previously described (17). All images are adjusted to similar brightness and contrast for comparison. The vessel analysis plugin program automatically measures the density and length density of retinal vasculature in the selected area. Vessels with diameters greater than 40 $\mu \mathrm{m}$ were classified as retinal arterioles and venules and were excluded from the capillary vasculature analysis. Furthermore, if any irregularity in tissue image from tissue folds or suboptimal staining was noted, these regions were excluded from the analysis.

Vascular density was defined as the percentage of area covered by vessels over the total selected area of interest. Vascular length density was defined as the percentage of total skeletonized vessel area over the total selected area of interest such that vessels with the same length would have the same vascular length density regardless of their width. A mean retinal vascular density and length density for each plexus layer of each study eye was obtained by averaging the findings for the four areas of interest.

ANOVA was used in the statistical analysis to determine whether the mean differences among the groups are statistically significant. Then post-hoc tests with Bonferroni
Cheung et al. CD $34^{+}$cells on capillary plexus in diabetic retinopathy

correction were used to identify which particular differences between pairs of means are significant. A P value $<0.05$ was considered statistically significant.

\section{Results}

\section{Retinal capillary plexus morphologies}

For each retina, four areas of interest were imaged as shown in Figure 1. Three distinct layers of retinal capillary plexus were visualized for each area of interest upon Z-axis scrolling of each stack of scans obtained using confocal microscopy. As shown in Figure 2, each capillary plexus layer showed a distinct capillary branching pattern and morphology. In the superficial capillary plexus (Figure $2 A$ ), retinal arterioles and venules are seen with capillaries branching off in a straight pattern and directly connecting with the underlying intermediate capillary plexus. In contrast, the capillary branching pattern for the intermediate capillary plexus appears curvy and wavy (Figure $2 B$ ). Some of the retinal arterioles and venules are still observable but appear smaller in appearance than those in the superficial plexus layer. Scrolling into the deeper layers in the stack, the deep capillary plexus is identified by the nest-like network of thin capillaries (Figure 2C). The retinal arterioles and venules are not readily seen in the deep plexus layer.

\section{Quantitative analysis of retinal vascular density and vascular length density}

The retinal flat mount immunohistochemistry was performed in a total of 42 eyes ( 9 eyes with human $\mathrm{CD} 34^{+}$BMSCs injection, 12 eyes with PBS injection, 21 contralateral untreated eyes). All were imaged using confocal microscopy. For our quantitative analysis of vascular density and length density, we identified the three layers of capillary plexus for each area of interest based on the morphologies described above. Eyes with suboptimal image quality (as defined in Methods) were excluded in the data analysis. Table 1 describes in detail the included and excluded eyes for each plexus layer and each study group. Over $64 \%$ of the acquired images for each plexus layer for each study group had good image quality for inclusion in the quantitative analysis. Figure 3 shows representative magnified confocal microscopy images of the retinal capillaries in each of the three retinal plexus layers for the right eye treated with human $\mathrm{CD} 34^{+} \mathrm{BMSCs}$ (Figure $3 A-3 C$ ) showing a denser appearing retinal capillary network when compared to the capillaries in the 

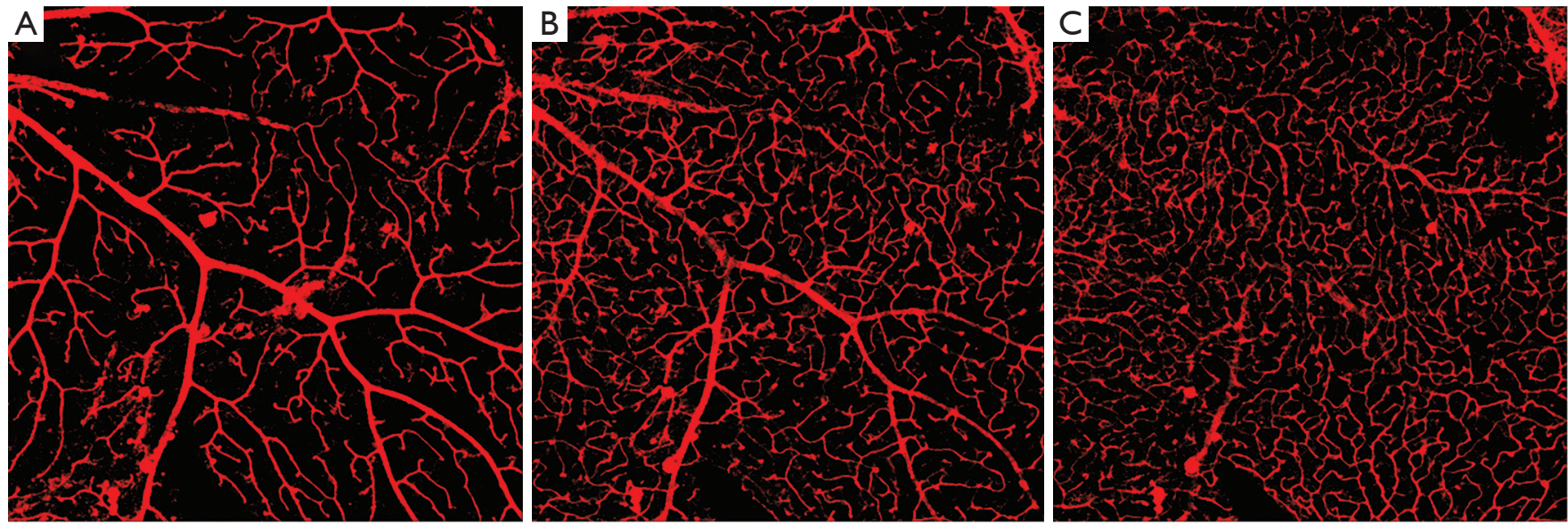

Figure 2 Retinal capillary plexus morphologies for the three plexus layers captured using confocal microscopy in retinal flat mount of diabetic mice following intravitreal injections of human $\mathrm{CD}_{3} 4^{+}$bone marrow stem cells. The blood vessels were stained using Isolectin-GSIB4. Representative image of retinal blood vessels in the superficial plexus (A), intermediate plexus (B), and deep plexus (C). Magnification, $\times 10$.

corresponding plexus layers of the right eye injected with PBS (Figure 3D-3F).

Quantitative analysis of retinal vascular density was performed for each of the three capillary plexus layers for each study group and summarized in Figure 4. In the superficial plexus, ANOVA showed that there was a statistically significant difference in the mean retinal vascular density among the four study groups $(\mathrm{P}=0.015)$. Post-hoc test with Bonferroni correction was used to compare the mean vascular density among the groups and human $\mathrm{CD}_{3} 4^{+} \mathrm{BMSCs}$ treated right eyes $\left[\mathrm{CD} 34^{+}(\mathrm{R})\right]$ had statistically significant higher mean vascular density than that of the contralateral untreated left eyes $\left[\mathrm{CD} 34^{+}(\mathrm{L})\right]$, PBS-treated right eyes $[\mathrm{PBS}(\mathrm{R})]$ and contralateral untreated left eyes $[\mathrm{PBS}(\mathrm{L})]$ with $\mathrm{P}$ values all $\leq 0.05$. In contrast, if the mean vascular density of the $\mathrm{PBS}(\mathrm{R})$ group was compared to that of the contralateral untreated left eye $[\mathrm{PBS}(\mathrm{L})]$, there was no significant difference with $\mathrm{P}$ values $>0.05$. Also, when the mean vascular density of the $\mathrm{CD} 34^{+}(\mathrm{L})$ group was compared to the PBS(L) group, no significant difference was noted ( $\mathrm{P}$ values $>0.05)$. An analysis of the intermediate and deep plexus shows a trend for the mean vascular density to be slightly higher in the $\mathrm{CD} 34^{+} \mathrm{BMSC}$ steated right eyes $\left[\mathrm{CD} 34^{+}(\mathrm{R})\right]$ when compared to the contralateral untreated left eyes $\left[\mathrm{CD} 34^{+}(\mathrm{L})\right]$, PBS-treated right eyes $[\mathrm{PBS}(\mathrm{R})]$ and contralateral untreated left eyes $[\mathrm{PBS}(\mathrm{L})]$ but the difference was not statistically significant ( $\mathrm{P}$ value $>0.05$ ).

Quantitative analysis of retinal vascular length density also was performed using ImageJ. The mean retinal vascular length density was compared among the study groups and summarized in Figure 5. The mean retinal vascular length density of the $\mathrm{CD}^{3} 4^{+}(\mathrm{R})$ group was significantly higher than that of $\mathrm{CD}_{3} 4^{+}(\mathrm{L}), \mathrm{PBS}(\mathrm{L})$, and $\mathrm{PBS}(\mathrm{R})$ for superficial capillary plexus layer (ANOVA post-hoc test $\mathrm{P}$ value $=0.01$ ). In contrast, the mean retinal vascular length density for the CD34 $(\mathrm{L})$ group was similar to that for PBS(L) and PBS(R) groups for the superficial plexus (ANOVA post-hoc test $\mathrm{P}$ values $>0.05$ ). For the intermediate and deep plexus layers, the mean retinal vascular length density of the $\mathrm{CD} 34^{+}(\mathrm{R})$ group was also higher than that of the other three groups, but the difference was not statistically significant.

\section{Discussion}

This study is a follow-up to our recent work showing a protective effect of intravitreal injection of human $\mathrm{CD} 34^{+}$BMSCs on the overall retinal vascular density in a murine model of diabetic retinopathy (10). Since diabetic retinopathy has been associated with retinal vascular changes in all three capillary plexus layers of the retina in human and murine eyes with diabetic retinopathy $(11-13,18)$, this study was conducted to determine whether the protective effects of the $\mathrm{CD} 34^{+} \mathrm{BMSC}$ can be observed in all three plexus layers of the retina following intravitreal administration. The study findings are important for evaluating the regenerative potential of this cell therapy and for understanding the potential mechanism of action of $\mathrm{CD} 34^{+}$cells in eyes with diabetic retinopathy 
Table 1 Summary of the number of samples and scans for each group

\begin{tabular}{|c|c|c|c|}
\hline Group & $\begin{array}{l}\text { Superficial } \\
\text { plexus }\end{array}$ & $\begin{array}{l}\text { Intermediate } \\
\text { plexus }\end{array}$ & Deep plexus \\
\hline \multicolumn{4}{|l|}{ (A) $\mathrm{CD}_{3} 4^{+}(\mathrm{R})$} \\
\hline Total number of scanned areas of interest & 36 & 36 & 36 \\
\hline Total number of scanned areas of interest included for quantitative analysis & $35(97.2 \%)$ & $29(80.6 \%)$ & $26(72.3 \%)$ \\
\hline \multicolumn{4}{|l|}{ (B) $\mathrm{CD}_{3} 4^{+}(\mathrm{L})$} \\
\hline Number of eyes with retinal flat mount & 9 & 9 & 9 \\
\hline Total number of scanned areas of interest & 36 & 36 & 36 \\
\hline Total number of scanned areas of interest included for quantitative analysis & $36(100 \%)$ & $32(88.9 \%)$ & $24(66.7 \%)$ \\
\hline \multicolumn{4}{|l|}{ (C) PBS(R) } \\
\hline Number of eyes with retinal flat mount & 12 & 12 & 12 \\
\hline Total number of scanned areas of interest & 48 & 48 & 48 \\
\hline $\begin{array}{l}\text { Number of scans excluded due to poor signal strength or tissue irregularity noted } \\
\text { in }>50 \% \text { of scan }\end{array}$ & $2(4.2 \%)$ & $9(18.8 \%)$ & $17(35.4 \%)$ \\
\hline Total number of scanned areas of interest included for quantitative analysis & $46(95.8 \%)$ & $39(81.2 \%)$ & $31(64.6 \%)$ \\
\hline \multicolumn{4}{|l|}{ (D) $\operatorname{PBS}(\mathrm{L})$} \\
\hline
\end{tabular}

(A) Right eyes that had intravitreal injections of human CD34 ${ }^{+}$BMSCs [CD34 $(R)$ ]; (B) contralateral non-injected left eyes in the CD34 ${ }^{+}$ group $\left[C D 34^{+}(\mathrm{L})\right]$; (C) right eyes that had intravitreal injections of PBS [PBS(R)]; (D) contralateral non-injected left eyes in the PBS group [PBS(L)]. BMSCs, bone marrow stem cells; PBS, phosphate-buffered saline.

Human $\mathrm{CD}_{3} 4^{+}$cells in bone marrow and peripheral blood have been explored as a potential regenerative therapy for both ischemic and nonischemic diseases (2). For eyes with retinal vasculopathy, prior studies have used animal models to show that human $\mathrm{CD} 34^{+}$cells from peripheral blood and bone marrow rapidly home into the damaged retinal vasculature following intravitreal injection with no longterm ocular or systemic adverse effects noted on histology $(5,9)$. Recently, our groups used in vivo retinal imaging and immunohistochemistry to demonstrate that the retinal homing of human CD $34^{+}$BMSCs following intravitreal injection is not limited to the retinal vasculature in a murine model of diabetic retinopathy (10). These human CD $34^{+}$ cells were found incorporated into the adjacent avascular retina as well but the retinal incorporation of these human cells was limited to the superficial layer of the retina. These observations raised a question whether the protective effects of $\mathrm{CD} 34^{+}$BMSCs after the intravitreal injection may be limited to the superficial retinal layer.

In our current study, we used immunohistochemistry and confocal microscopy to visualize the retinal vasculature at various retinal depths and characterize the effects of 

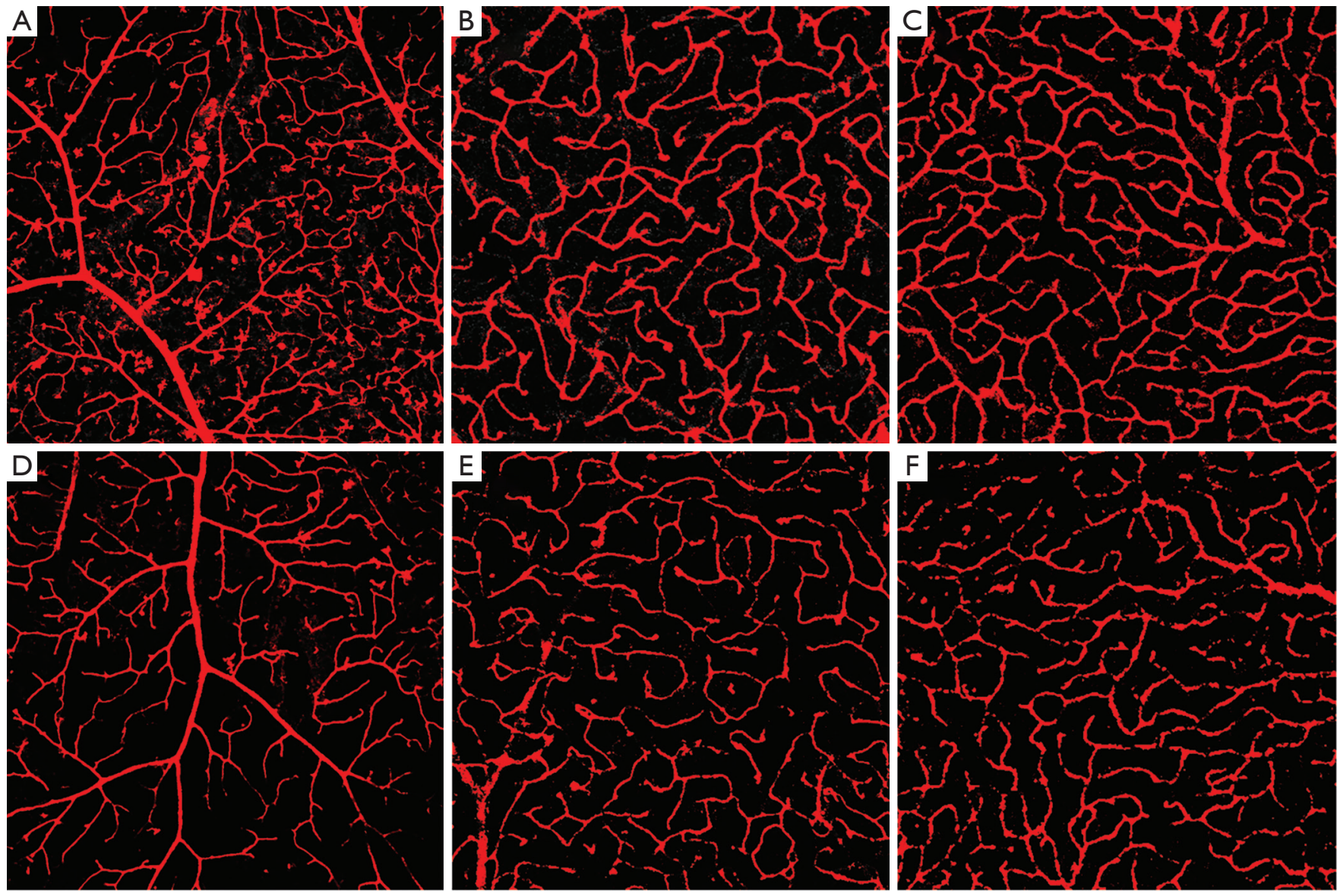

Figure 3 Confocal microscopy images of the retinal capillaries in the three retinal plexus layers comparing eyes following intravitreal CD $34^{+}$ BMSCs injections to eyes following intravitreal PBS injections. Images of the retinal vasculature obtained from retinal flat mount after staining with Isolectin-GS-IB4. (A-C) Representative image of retinal capillaries in the superficial plexus (A), intermediate plexus (B), and deep plexus (C) of the right eye 4 weeks following intravitreal CD34 $4^{+}$BSCs injection. (D-F) Representative image of retinal blood vessels in the superficial plexus (D), intermediate plexus (E), and deep plexus (F) of the right eye 4 weeks following intravitreal PBS injection. BMSCs, bone marrow stem cells; PBS, phosphate-buffered saline. Magnification: $(A, D) \times 12$; (B-F) $\times 20$.

intravitreal injection of human $\mathrm{CD} 34^{+} \mathrm{BMSCs}$ on the three retinal capillary plexus layers in murine eyes with diabetic retinopathy. The retinal flat-mount and retinal tissue immunostaining were performed in eyes collected 4 weeks after the intravitreal injection of human $\mathrm{CD} 34^{+}$BMSCs, i.e., 6 months after induction of diabetes. This time point was selected since the Alzet pump does not deliver the immunosuppressive drugs effectively thereafter and the mice poorly tolerated longer systemic immunosuppressive therapy. Our prior study using in vivo retinal imaging demonstrated development of early retinal microvascular changes, i.e., retinal ischemia and fluorescein leakage, consistent with diabetic retinopathy between 5 and
6 months after induction of diabetes in this animal model (10).

Our study showed that confocal microscopy can be used to visualize the three retinal capillary plexuses (superficial, intermediate, and deep plexus) in retinal flat mount tissue after immunostaining of the vasculature. Each capillary plexus layer had a distinct morphology of branching capillaries (Figure 2). Eyes with significant processing artifacts, such as tissue folding or irregular immunostaining were excluded from the study quantitative analysis.

Our study used ImageJ analysis to quantitate the retinal vascular density and vascular length density of each capillary plexus layer to determine the effects of $\mathrm{CD} 34^{+}$BMSCs on 
A
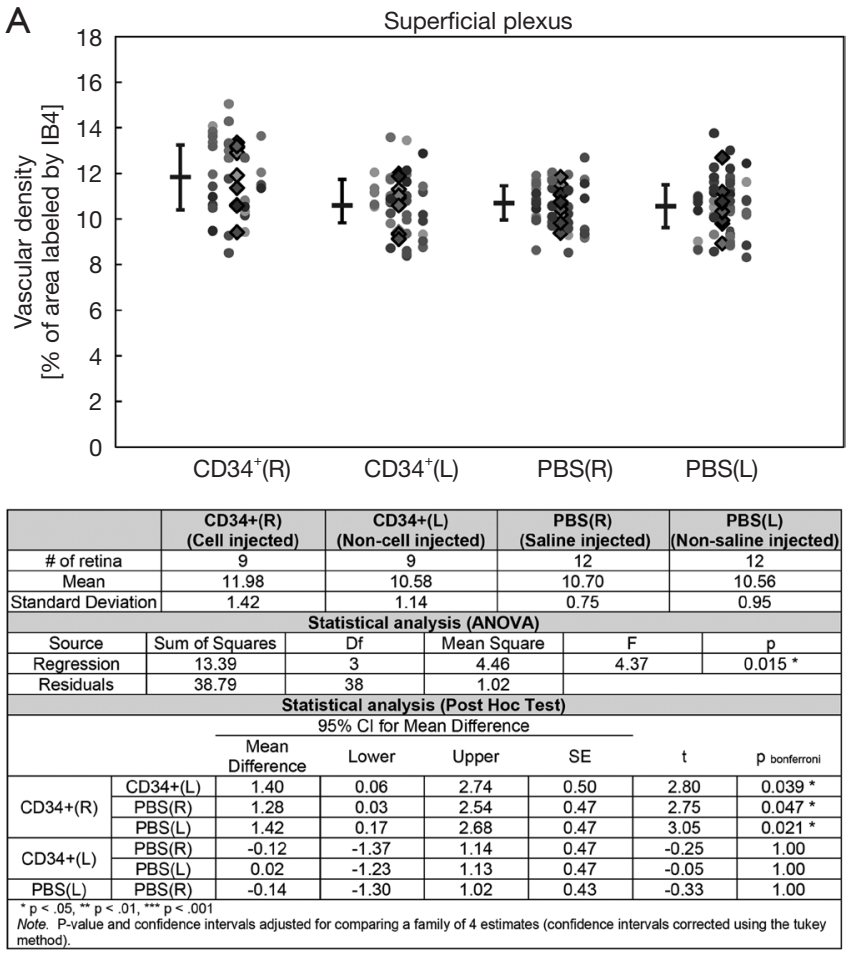

c
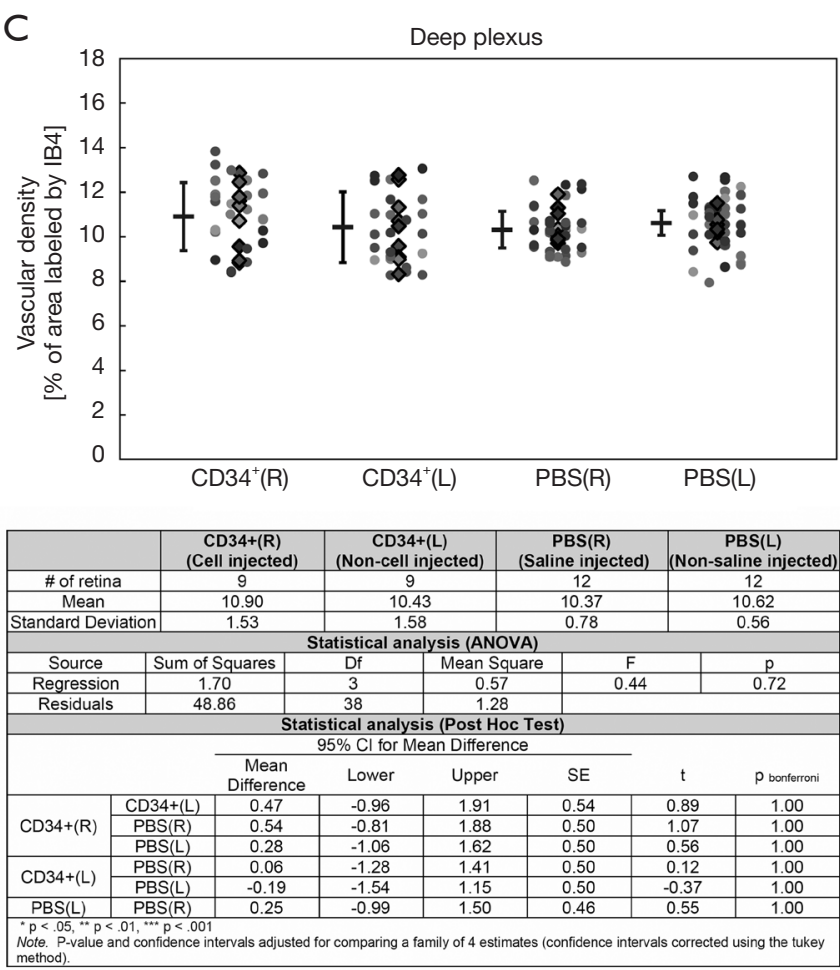

B $18 \quad$ Intermediate plexus
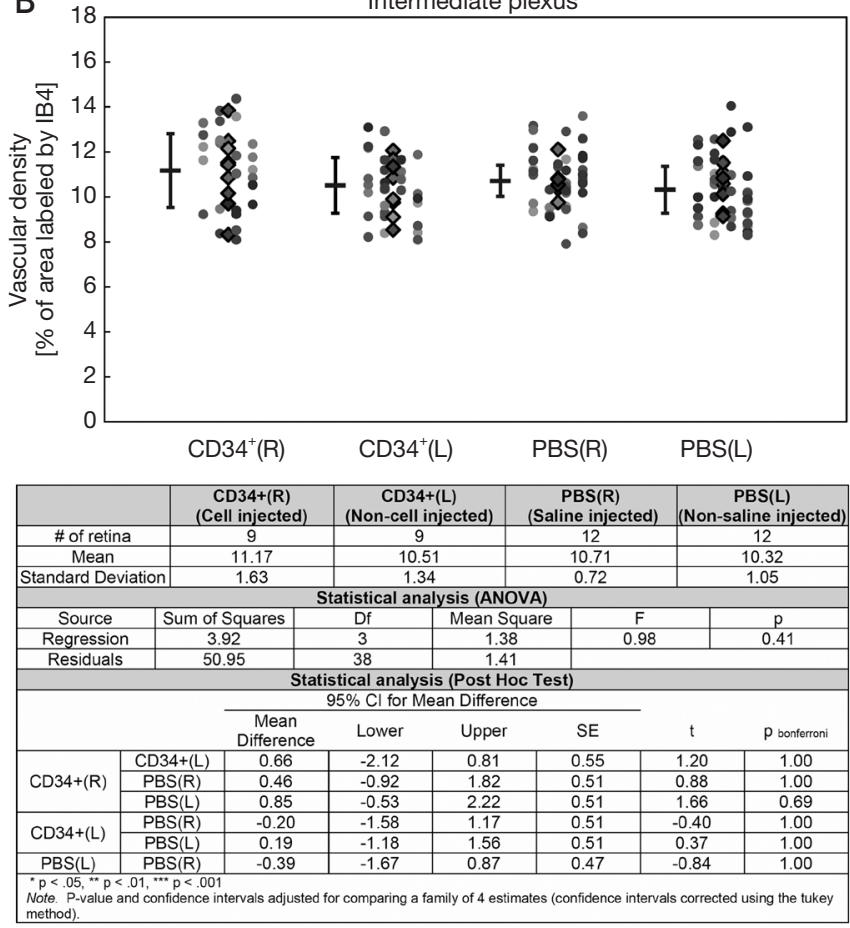

Figure 4 Quantification of retinal vascular density (expressed as the percentage of vessel area in the selected area of the plexus). Blood vessels in retinal flat mount were stained with Isolectin-GS-IB4 and imaged using confocal microscopy. Vascular density was obtained using ImageJ. Statistical analysis (ANOVA and post-hoc tests) performed for each selected plexus layers using SuperPlots. (A) Vascular density of the superficial plexus. (B) Vascular density of the intermediate plexus. (C) Vascular density of the deep plexus. PBS, phosphate-buffered saline; R, right; L, left. 

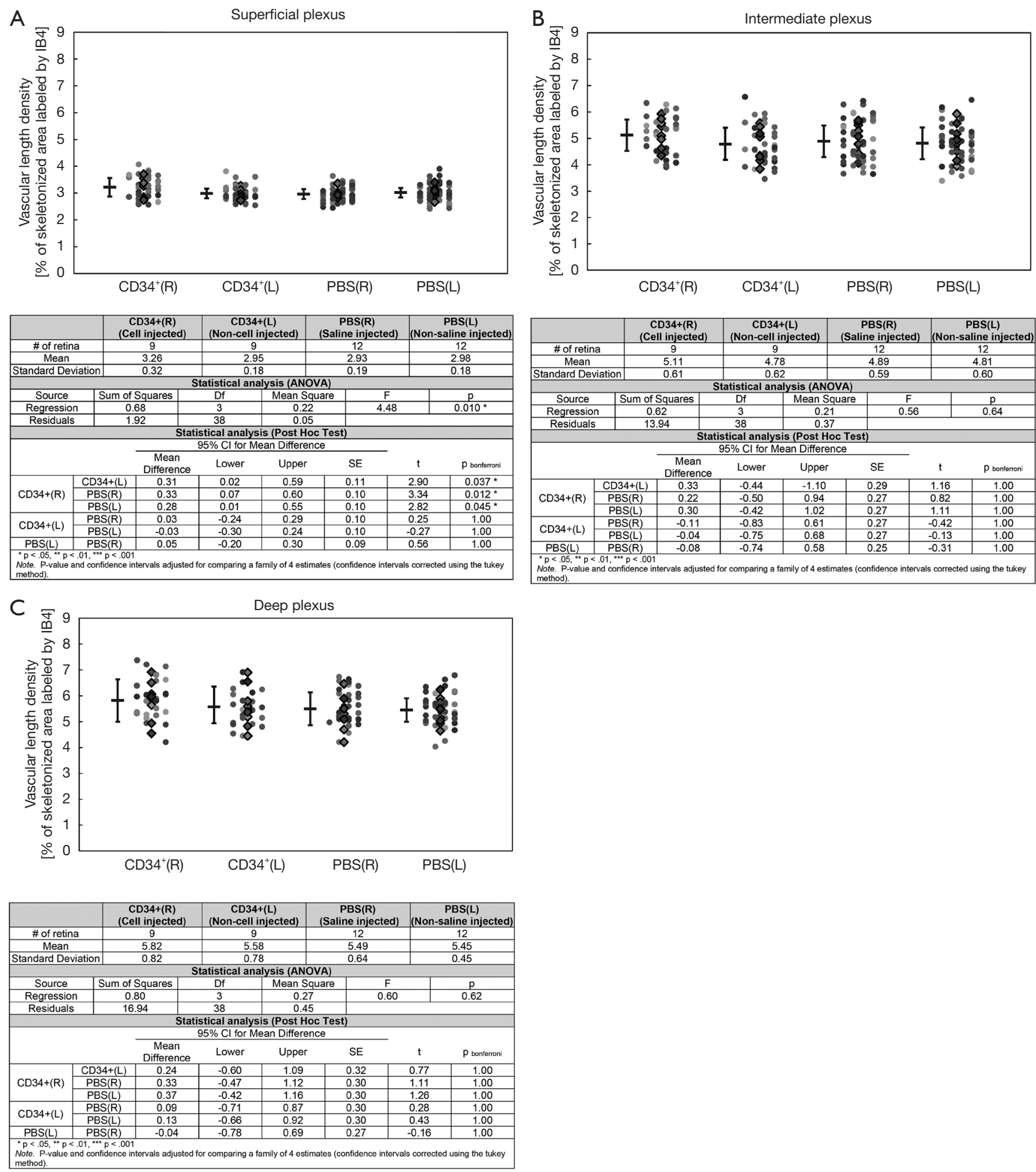

Figure 5 Quantification of retinal vascular length density (expressed as the percentage of skeletonized vessel area in the selected area of the plexus). Blood vessels in retinal flat mount were stained with Isolectin-GS-IB4 and imaged using confocal microscopy. Vascular length density was obtained using ImageJ. Statistical analysis (ANOVA and post-hoc tests) performed for each selected plexus layers using SuperPlots. (A) Vascular length of the superficial plexus. (B) Vascular length of the intermediate plexus. (C) Vascular length of the deep plexus. PBS, phosphate-buffered saline; R, right; L, left. 
retinal vascular health in each of these plexus layers in eyes with diabetic retinopathy. This is important since retinal vascular changes associated with diabetic retinopathy have been observed in all three plexus layers $(11-13,18)$. Confocal microscopy has been used previously in the murine retina with diabetic retinopathy demonstrating retinal vascular changes in all three plexus layers with the earliest changes being observed in the deep plexus $(18,19)$. Similarly, in human eyes with diabetic retinopathy, a recent cohort study has shown that the degree of capillary loss in the deep retinal vascular plexus due to diabetic retinopathy correlated strongest with the decrease in visual acuity in patients with severe non-proliferative or proliferative diabetic retinopathy although the correlation was also noted for changes in the superficial and intermediate plexus layers (13). Furthermore, changes in vascular density in the intermediate and deep plexus appeared to be associated with disease progression (11). These human studies were performed using in vivo retinal imaging, i.e., optical coherence tomography angiography.

In our study, we recently reported that murine eyes with diabetic retinopathy injected with human $\mathrm{CD} 34^{+}$BMSCs have higher vascular density when compared to PBS treated eyes (10). Detailed confocal microscopy analysis of the three retinal plexus layers conducted in our current study demonstrates an increase in retinal vascular density and length density which was significant for the superficial plexus layer. For the intermediate and deep plexus layers, the mean retinal vascular density and length density tended to be higher for the CD34 $4^{+}$BMSC injected eyes when compared to untreated or PBS treated groups, but the difference was not statistically significant. The findings support the hypothesis that $\mathrm{CD} 34^{+} \mathrm{BMSC}$ have protective effects on the retinal vasculature in eyes with diabetic retinopathy. However, the protective effects on the deeper vascular plexus layers of retina may not be as pronounced as the effects on the superficial retina. Since our prior immunohistochemical analysis of the retina identified human $\mathrm{CD} 34^{+}$cells only in the superficial layers of the retina following intravitreal injection (10), the reduced penetration and engraftment of the $\mathrm{CD} 34^{+}$cells into the deeper layers of the retina may limit the regenerative effect of these cells in the deeper retinal vascular layers. This observation is noteworthy since the protective effects of the $\mathrm{CD} 34^{+}$cells on the retina has been attributed at least in part to paracrine effects of these cells since the protective effects of bone marrow hematopoietic stem cells following intravitreal injection was observed in eyes with retinal degeneration without direct engraftment of these cells in the degenerating photoreceptor layer (2).

Our study has several limitations. First, even though the STZ-induced murine model is a commonly used animal model for diabetic retinopathy, it does not fully replicate human disease. For example, retinal neovascularization and retinal neuronal degeneration have not been observed in this model (14). Thus, future studies using alternate animal models of diabetic retinopathy may be helpful to fully elucidate the effects of $\mathrm{CD} 34^{+}$cells in eyes with diabetic retinopathy. Second, the confocal microscopy analysis was limited to retina within $1 \mathrm{~mm}$ from the optic disc. This was done to limit the time required to acquire scanned images and avoid imaging the far peripheral retina where fixation artifacts tend to occur. Nonetheless, it is of note that the observed changes in retinal vascular density and length in the superficial retinal vascular plexus layer parallel the changes observed in our prior work where the entire retinal area and thickness were analyzed in total (10). Although our current study did not demonstrate a significant protective effect of intravitreal injection of human $\mathrm{CD} 34^{+} \mathrm{BMSCs}$ on the intermediate and deep plexus layers of the retina, it is important to note that we had a limited sample size per study group. Our study may not have been powered enough to detect more subtle effects of the CD $34^{+}$BMSCs on these deeper retinal vascular layers. Lastly, there were some tissue preparation artifacts noted in some eyes from the flat-mount process or immunohistochemistry. These artifacts included retinal folds, retinal tears, and areas of irregular immunostaining. To minimize the effect of these artifacts on our quantitative analysis, we limited our analysis to eyes with good image acquisition signal on confocal microscopy and eyes where these processing artifacts were present in less than $50 \%$ of the retinal area being imaged. As a result, approximately $15.7 \%$ (79/504) of scanned images for the different capillary plexus layers were excluded.

In conclusion, intravitreal injection of human CD $34^{+}$ BMSCs resulted in a significantly higher retinal vascular density and length density in the superficial retinal capillary plexus layer in this murine model of diabetic retinopathy. The findings support the hypothesis that intravitreal injection of human $\mathrm{CD} 34^{+} \mathrm{BMSCs}$ has potential protective effects on the retinal vasculature in eyes with diabetic retinopathy. The lack of observed significant protective effects of these cells on the deeper retinal vascular layers suggests that direct engraftment of the $\mathrm{CD} 34^{+}$cells in the affected retinal layer may be important in achieving the full 
protective effects of these cells in the retinal vasculature. Since $\mathrm{CD} 4^{+}$cells from peripheral blood of a diabetic human have been shown to have defective retinal vascular homing (5), the development of a method to enhance the retinal engraftment of these $\mathrm{CD} 34^{+}$cells may be an important step in maximizing the regenerative potential of this cell therapy on the retina. An early-phase clinical trial has been conducted showing the feasibility and safety of intravitreal injection of autologous $\mathrm{CD}_{3} 4^{+} \mathrm{BMSC}$ in eyes with retinal ischemia or degeneration (20). A larger phase I/II clinical trial has been started exploring the safety and potential efficacy of intravitreal autologous CD34 ${ }^{+}$BMSC therapy in eyes with vision loss from central retinal vein occlusion, another common retinal vascular cause of vision loss (www.clinicaltrial.gov NCT03981549). As we gain more preclinical and clinical information regarding the effects of human $\mathrm{CD}_{3} 4^{+}$cells on the retina, the potential of using intravitreal injection human $\mathrm{CD} 34^{+} \mathrm{BMSCs}$ to treat eyes with vision loss from diabetic retinopathy can be explored in future larger clinical trials.

\section{Acknowledgments}

The authors thank Dr. Qizhi Gong for her assistance in operating the confocal microscope and Joel Misefeld for demonstrating the process of retinal extraction, dissection, and flat-mount.

Funding: This research was supported in part by the J. William Kohl, M.D. Research Scholarship for Medical Students from the Department of Ophthalmology \& Vision Science of the University of California, Davis (KWC). and the University of California Davis Eye Center Retina Research Fund (SSP).

\section{Footnote}

Provenance and Peer Review: This article was commissioned by the editorial office, Annals of Translational Medicine for the series "Novel Tools and Therapies for Ocular Regeneration". The article has undergone external peer review.

Reporting Checklist: The authors have completed the ARRIVE reporting checklist. Available at https://dx.doi. org/10.21037/atm-20-3930

Data Sharing Statement: Available at https://dx.doi. org/10.21037/atm-20-3930
Peer Review File: Available at https://dx.doi.org/10.21037/ atm-20-3930

Conflicts of Interest: All authors have completed the ICMJE uniform disclosure form (available at https://dx.doi. org/10.21037/atm-20-3930). The series "Novel Tools and Therapies for Ocular Regeneration” was commissioned by the editorial office without any funding or sponsorship. Susanna S. Park served as the unpaid Guest Editor of the series and serves as an unpaid editorial board member of Annals of Translational Medicine from Oct 2019 to Sep 2021, and reports grants from Allergan, grants from Roche/ Novartis, outside the submitted work. The authors have no other conflicts of interest to declare.

Ethical Statement: the authors are accountable for all aspects of the work in ensuring that questions related to the accuracy or integrity of any part of the work are appropriately investigated and resolved. All experiments in this study were conducted in accordance with the Association for Research in Vision and Ophthalmology (ARVO) Statement for the Use of Animals in Ophthalmic and Vision Research and a protocol approved by the Institutional Animal Care and Use Committee at the University of California Davis mandated by the Animal Welfare Act and the Healthy Research Extension Act (Protocol \#20162).

Open Access Statement: This is an Open Access article distributed in accordance with the Creative Commons Attribution-NonCommercial-NoDerivs 4.0 International License (CC BY-NC-ND 4.0), which permits the noncommercial replication and distribution of the article with the strict proviso that no changes or edits are made and the original work is properly cited (including links to both the formal publication through the relevant DOI and the license). See: https://creativecommons.org/licenses/by-nc-nd/4.0/.

\section{References}

1. Cheung N, Mitchell P, Wong, TY, et al. Diabetic retinopathy. Lancet 2010;376:124-36.

2. Park SS, Moisseiev E, Bauer G, et al. Advances in bone marrow stem cell therapy to treat retinal dysfunction. Prog Retin Eye Res 2017;56:148-65.

3. Harraz M, Jiao C, Hanlon HD, et al. CD34- bloodderived human endothelial cell progenitors. Stem Cells 2001;19:304-12. 
4. Asahara T, Murohara T, Sullivan A, et al. Isolation of putative progenitor endothelial cells for angiogenesis. Science 1997;275:964-7.

5. Caballero S, Sengupta N, Afzal A, et al. Ischemic vascular damage can be repaired by healthy, but not diabetic endothelial progenitor cells. Diabetes 2007;56:960-7.

6. Mackie AR, Losordo DW. CD34-positive stem cells: in the treatment of heart and vascular disease in human beings. Tex Heart Inst J 2011;38:474-85.

7. Rafii S, Lyden D. Therapeutic stem and progenitor cell transplantation for organ vascularization and regeneration. Nat Med 2003;9:702-12.

8. Caballero S, Hazra S, Bhatwadekar A, et al. Circulating mononuclear progenitor cells: differential roles for subpopulations in repair of retinal vascular injury. Invest Ophthalmol Vis Sci 2013;54:3000-9.

9. Park SS, Caballero S, Bauer G, et al. Long-term effects of intravitreal injection of GMP-grade bone marrowderived CD34+ cells in NOD-SCID mice with acute ischemia-reperfusion injury. Invest Ophthalmol Vis Sci 2012;53:986-94

10. Yazdanyar A, Zhang P, Dolf C, et al. Effects of intravitreal injection of human CD34+ bone marrow stem cells in a murine model of diabetic retinopathy. Exp Eye Res 2020;190:107865.

11. Onishi AC, Nesper PL, Roberts PK, et al. Importance of considering the middle capillary plexus on OCT angiography in diabetic retinopathy. Invest Ophthalmol Vis Sci 2018;59:2167-76.

12. Park JJ, Chung CS, Fawzi AA. Visualizing Structure and Vascular Interactions: Macular Nonperfusion in Three Capillary Plexuses. Ophthalmic Surg Lasers Imaging

Cite this article as: Cheung KW, Yazdanyar A, Dolf C, Cary W, Marsh-Armstrong N, Nolta JA, Park SS. Analysis of the retinal capillary plexus layers in a murine model with diabetic retinopathy: effect of intravitreal injection of human CD34 ${ }^{+}$ bone marrow stem cells. Ann Transl Med 2021;9(15):1273. doi: 10.21037/atm-20-3930
Retina 2018;49:e182-e190.

13. Dupas B, Minvielle W, Bonnin S, et al. Association Between Vessel Density and Visual Acuity in Patients With Diabetic Retinopathy and Poorly Controlled Type 1 Diabetes. JAMA Ophthalmol 2018;136:721-28.

14. Feit-Leichman RA, Kinouchi R, Takeda M, et al. Vascular damage in a mouse model of diabetic retinopathy: relation to neuronal and glial changes. Invest Ophthalmol Vis Sci 2005;46:4281-7.

15. Moisseiev E, Smit-McBride Z, Oltjen S, et al. Intravitreal administration of human bone marrow CD34+ Stem cells in a murine model of Retinal Degeneration. Invest Ophthalmol Vis Sci 2016;57:4125-35.

16. Soto I, Oglesby E, Buckingham BP, et al. Retinal ganglion cells downregulate gene expression and lose their axons within the optic nerve head in a mouse glaucoma model. J Neurosci 2008;28:548-61.

17. Zarb Y, Weber-Stedlbauer U, Kirshenbaum D, et al. Ossified blood vessels in primary familial brain calcification elicit a neurotoxic astrocyte response. Brain 2019;142:885-902.

18. McLenachan S, Magna AL, Ramos D, et al. Angiography reveals novel features of the retinal vasculature in healthy and diabetic mice. Exp Eye Res 2015;138:6-21.

19. Piano I, Novelli E, Della Santina L, et al. Involvement of autophagic pathway in the progression of retinal degeneration in a mouse model of diabetes. Front Cell Neurosci 2016;10:42.

20. Park SS, Bauer G, Abedi M, et al. Intravitreal autologous bone marrow-derived CD34+ cells for ischemic and degenerative retinal disorders: preliminary phase 1 clinical trial findings. Invest Ophthalmol Vis Sci 2014;56:81-9. 